\title{
Propos des musiciens montagnards du Beskide silésien (Pologne)
}

Remarks by the musicians of the Beskydy mountains in the Silesian region of Poland

\section{Aurélia Domaradzka Barbier}

\section{OpenEdition}

\section{Journals}

Édition électronique

URL : http://journals.openedition.org/ethnomusicologie/1827

ISSN : 2235-7688

\section{Éditeur}

ADEM - Ateliers d'ethnomusicologie

\section{Édition imprimée}

Date de publication : 1 janvier 1998

Pagination : 87-106

ISBN : 2-8257-0639-6

ISSN : 1662-372X

\section{Référence électronique}

Aurélia Domaradzka Barbier, "Propos des musiciens montagnards du Beskide silésien (Pologne) », Cahiers d'ethnomusicologie [En ligne], 11 | 1998, mis en ligne le 26 mars 2012, consulté le 20 avril 2019. URL : http://journals.openedition.org/ethnomusicologie/1827

Ce document a été généré automatiquement le 20 avril 2019

Tous droits réservés 


\title{
Propos des musiciens montagnards du Beskide silésien (Pologne)
}

\author{
Remarks by the musicians of the Beskydy mountains in the Silesian region of \\ Poland
}

Aurélia Domaradzka Barbier

1 En Europe Centrale, le cas de la Pologne, et de la Silésie en particulier, est sur le plan scientifique extrêmement intéressant et, en quelque sorte, exemplaire. En effet, la Pologne est située entre deux zones de fracture de l'Europe, l'une à l'ouest du pays entre les mondes germanique et slave et l'autre à l'est du pays entre les mondes catholique et orthodoxe. La Silésie, quant à elle, a longtemps été un enjeu entre les Polonais, les Tchèques et les Allemands, y compris au plan des influences culturelles. De plus, à partir de 1945, des déplacements de populations de la Silésie vers l'Allemagne et de toutes les régions de Pologne vers la Silésie ayant été organisés pour des raisons politiques et économiques, des évolutions culturelles importantes ont eu lieu. Il y a là de vastes perspectives pour la recherche (Skrzypczak 1996). Mais le concept de «nouvelle histoire » dégagée de préoccupations nationalistes ou idéologiques, par exemple pour l'histoire des nationalités ayant vécu ou vivant en Silésie, n'existe en Pologne que depuis quelques années et presque tout reste à faire.

2 La Silésie est aujourd'hui une région entièrement polonaise située dans le sud-ouest du pays. On y distingue une zone de plaine au nord, la vallée de l'oder et une zone montagneuse au sud: les Sudètes et le Beskide silésien. Ce dernier, situé dans l'extrême sud de la région, fait partie de la chaîne des Carpates. Dans le district de Cieszyn, non loin de Wisla, il existe un endroit nommé par la population locale «trojkat beskidzki » c'est-àdire «le triangle du Beskide». Sous cette appellation se cachent trois villages: Istebna, Koniakow et Jaworzynka, ainsi que des hameaux environnants. Reliés entre eux par des vallées, ils forment sur la carte un triangle, d'où leur nom. 


\section{Le point sur la recherche ethnomusicologique en Silésie}

Compte tenu de la spécificité et de la richesse folklorique de la Silésie, les collectes de chants réalisées depuis plus d'un siècle et demi par des amateurs et des ethnologues y ont été considérables. Elles se sont soldées, jusqu'à un passé récent, par de nombreuses publications de recueils de chants qui devaient prouver, déjà par leur simple existence, et souvent par leurs analyses sociologiques, «la polonité » de la Silésie, selon les objectifs politiques de l'époque. Ce procédé était d'autant plus discutable que beaucoup de Silésiens ne reconnaissaient pleinement leur appartenance culturelle ni à la Pologne ni à l'Allemagne, soulignant ainsi leur particularisme. Dans une étude récente, nous avons proposé de nouvelles hypothèses concernant l'évolution du chant silésien. A côté du travail de terrain, environ trois cent quatre vingt références bibliographiques et discographiques y ont été prises en compte, dont mille cinq cent chants avec paroles (Domaradzka 1997 : 907-935). Mais les musiques des montagnards du Beskide silésien sont pratiquement absentes de ces références bibliographiques, car le petit territoire situé dans l'extrême sud de la région n'a pas suscité beaucoup d'intérêt chez les chercheurs. Déjà en 1938 on s'étonnait que les chants d'Istebna n'aient pas encore été étudiés, et cela malgré leur richesse (Preizner 1938: 30). Mises à part des informations sur les chants locaux notées par quelques autochtones, souvent instituteurs de villages (Sikora 1993 : 27-42), il n'existe à notre connaissance que deux publications importantes concernant « le triangle du Beskide » (Tacina 1939 et Kopoczek 1984). Notre travail a évidemment pris en compte ces deux publications. Mais il est difficile de résumer les neuf cent soixante-sept pages d'une thèse analysant le folklore musical silésien à plusieurs niveaux de discours verbal et musical dans son contexte, intégrant aussi les avancées de l'ethnomusicologie locale. De plus, la quasi totalité des publications prises en compte est malheureusement en langue polonaise, très peu lue en Occident. Enfin, les dernières publications silésiennes en la matière s'arrêtent à l'année 1984, car dans le pays « en état de guerre » déclaré en décembre 1981 par le Général Jaruzelski, il y avait d'autres préoccupations que le folklore. Néanmoins, les choses ont changé à partir de 1993. Dans la Pologne libérale et en pleine expansion économique, les nouveaux articles définissent surtout les tâches confiées à l'ethnomusicologie. 
Fig. 1 : Le Beskide silésien

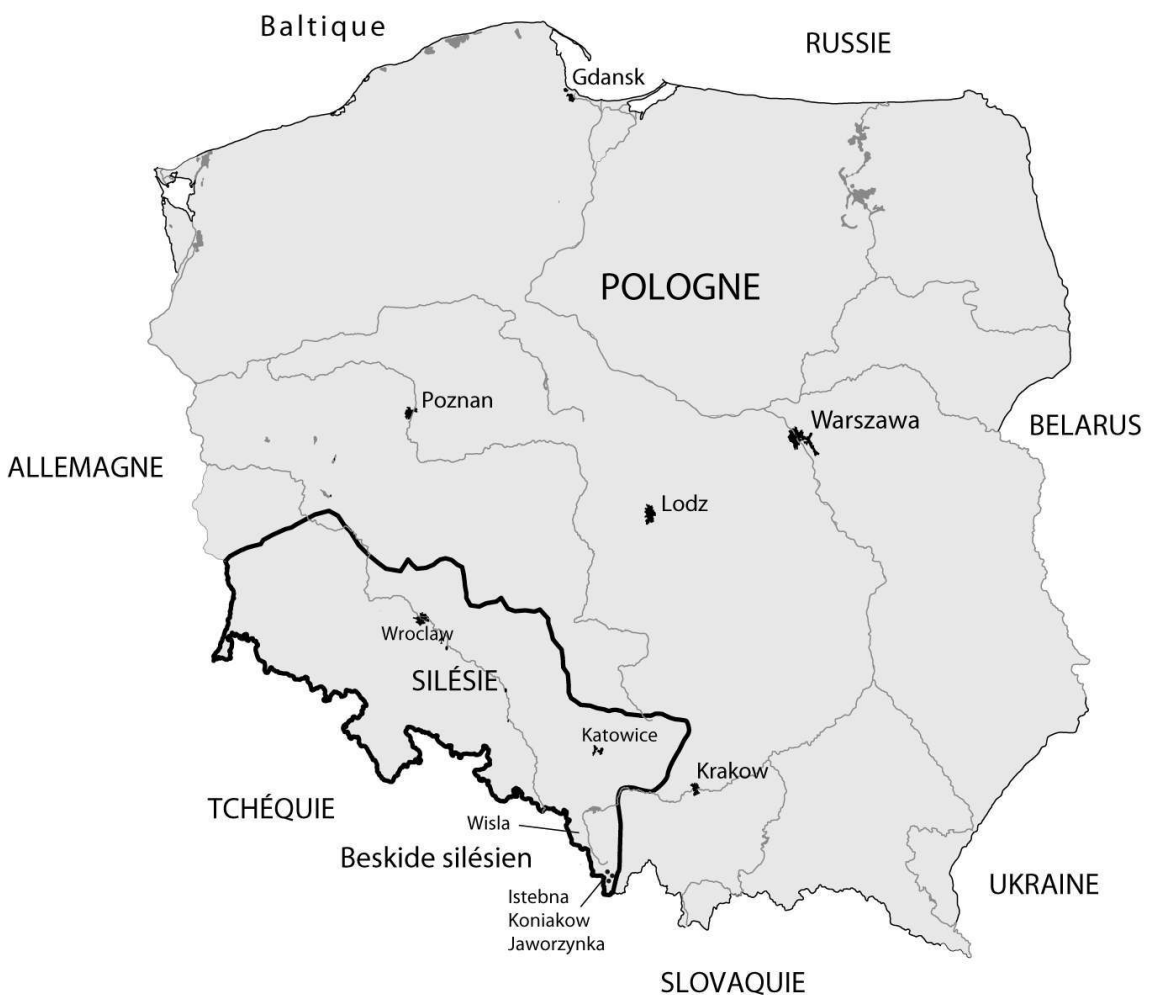

\section{Le travail sur le terrain dans le Beskide silésien}

4 Nos recherches en Silésie se sont échelonnées entre 1990 et 1995, mais notre travail sur le terrain dans le Beskide silésien s'est surtout déroulé entre 1993 et 1994.

5 Notre étude consacrée aux chants silésiens a été axée d'avantage sur les aspects musicologiques qu'ethnologiques. Néanmoins, nous souhaitons proposer dans cet article une contribution modeste, mais unique, concernant les propos des montagnards silésiens sur leur musique. En effet, nous avons vécu en Silésie pendant dix-neuf ans. Imprégnée de sa culture et connaissant sa langue, puis l'ayant quittée en 1984, il nous a paru souhaitable d'y revenir pour y observer la vie locale, avec l'avantage d'être à la fois de la région et étrangère à la région. Pour constituer une importante phonothèque de musiques silésiennes, nous avons rencontré de nombreux musiciens et avons ainsi pu être le témoin non seulement de leurs productions musicales, mais aussi de leurs propos sur la musique.

\section{La méthodologie de l'enquête sur le terrain}

6 Dans la collecte sur le terrain des informations utiles pour une exploration scientifique, en l'absence de sources formelles et bien reconnues, se pose le problème de la fiabilité des informations recueillies. Devant cette situation, nous avons délibérément pris la décision d'interroger un certain nombre d'autochtones soigneusement sélectionnés, s'assurant de la fiabilité de leurs informations. Les informateurs ayant contribué de façon significative à cette enquête ont été : Zuzanna Kawulok, fondatrice d'un petit musée d'instruments 
traditionnels, Zbigniew Walach, agriculteur, Monika Bocek Walach, femme au foyer, Czeslaw Weglarz, agriculteur, Jacek Kotarba, étudiant, Jozef Broda, instituteur, Cezary Drzewiecki, organisateur de la Semaine de la Culture du Beskide.

7 Pour comprendre leurs propos, la description des contextes dans lesquels nos informateurs évoluent et ont évolué s'impose. Nous avons fait trois types de rencontres: individuelles au domicile des musiciens, collectives en plein air et dans les fermes avec des musiciens, et collectives avant et après leurs passages sur des scènes montées en été dans plusieurs localités voisines de Wisla. Ces représentations sont organisées chaque année, au mois d'août, sous la présidence de la Ville de Wisla, dans le cadre de la Semaine de la Culture du Beskide.

8 Nous avons ainsi pu rencontrer plusieurs personnes intéressantes, parmi lesquelles il y avait des musiciens extrêmement actifs et très appréciés par la population locale. Il s'agit d'abord de Zuzanna Kawulok, la cinquantaine, fille du célèbre instrumentiste traditionnel Jan Kawulok, aujourd'hui décédé, et qui habite Istebna. Sa famille vit de la terre et de l'élevage, mais elle se fait aussi un appoint en accueillant chez elle des touristes. Dans une partie de sa maison, elle a organisé un «petit musée » d'instruments de musique. Elle a hérité des instruments traditionnels de son père Jan Kawulok, qui les construisait luimême et savait très bien en jouer. En 1976, il était même capable de réaliser une fujarka, sorte de flûte à huit tons, sur un modèle d'il y a 400 ans trouvé à Ostrowek en Silésie d'Opole. L'instrument de prédilection de Zuzanna Kawulok est la cornemuse nommée gajdy. Mais elle aime aussi raconter en s'accompagnant du piszczek, une sorte d'appeau, et faire de la musique d'ensemble avec les enfants du village (aux flûtes piszczalki et fujarki). Ses informations sur les coutumes locales, sur les préoccupations quotidiennes des habitants du village et sur les instruments de musique ont été précieuses. Un autre informateur, Zbigniew Walach, habitant aussi Istebna, fait de la musique d'ensemble avec sa famille et ses amis : Monika Bocek-Walach, Czeslaw Weglarz et Jacek Kotarba. Ils sont surtout instrumentistes et chanteurs. Ils jouent, entre autres, du gajdy, du zlobcoki (violon traditionnel), du violon, de la contrebasse et des ocarinas qu'ils fabriquent eux-mêmes. Avec leurs familles, ils vivent essentiellement de la terre. Mais après le travail, Zbigniew Walach dirige aussi des orchestres de jeunes de la région en les initiant à l'art instrumental. Un autre "personnage clef», Jozef Broda de Koniakow dans le Beskide silésien, instituteur à l'école communale, donne libre cours à son imagination en construisant des piszczalki qui servent de matériel pédagogique pour ses élèves. Il considère que sa participation active aux manifestations d'été est indispensable. Par ailleurs, les contacts avec la population locale et avec Cezary Drzewiecki, directeur des Semaines de la Culture du Beskide, lors des manifestations organisées initialement à Wisla et étendues par la suite à d'autres villages voisins, nous ont permis de mieux comprendre les motivations qui poussent les autochtones à participer à ce genre de manifestations.

9 Mises à part ces rencontres estivales qui constituent une ouverture sur le monde extérieur, il existe encore des coutumes familiales et saisonnières pratiquées dans les villages, à certains moments de l'année, dans lesquelles la musique trouve sa place.

\section{Les pratiques musicales des montagnards silésiens}

10 Afin d'analyser les propos des musiciens montagnards silésiens sur leurs pratiques musicales et le cadre dans lequel elles s'inscrivent, nous traiterons la problématique selon 
trois approches : leur origine, leur cadre de vie ainsi que les motivations et les moyens de ces musiciens. Dans ce contexte, l'analyste doit chercher à répondre aux sept questions suivantes :

Quelle est l'identité des montagnards silésiens et qu'en disent-ils?

Quelle est la façon de vivre des montagnards silésiens?

Que disent les musiciens montagnards sur leurs motivations et leurs moyens?

C'est à dire :

Pourquoi font-ils de la musique?

Comment font-ils de la musique?

Que chantent-ils?

Que disent-ils de leur musique?

\section{L'identité des montagnards silésiens}

11 La notion d'identité chez les montagnards silésiens nécessite une brève présentation de leurs origines géographiques et de leur positionnement ethnique et culturel. Les montagnards du Beskide silésien ressentent un besoin de parler d'eux-mêmes, un besoin de se définir, de se situer dans la société et de montrer leur particularisme. Cette quête des origines remonte très loin dans le temps et elle se transmet toujours dans la famille. Ils ont leur identité propre car, avant d'être polonais ou silésiens, ils sont avant tout montagnards. Selon Zbigniew Walach, ils sont d'abord un peuple pastoral descendant des Valaques venus de l'est qui se sont mélangés à la population locale - il en parle toujours en utilisant le terme "nous». Le nom de Valaque (Walach à Istebna) est pour eux synonyme de peuple berger ${ }^{1}$ et encore aujourd'hui les montagnards se nomment les Valaques. Cela correspond aussi au nom de famille de Zbigniew. Les montagnards, musiciens ou non, expriment également leur singularité dans le domaine vestimentaire car, à côté du costume de montagnard silésien, on porte dans la région une tenue traditionnelle spécifiquement valaque. Mais il faut savoir que le costume valaque caractérisant toute la Silésie de Cieszyn dont le Beskide silésien fait partie, était à l'origine celui de la bourgeoisie locale. Arrivé ici au début du XVIIIe siècle, il s'est diffusé ensuite dans les milieux d'ouvriers et d'artisans. Il n'arrive chez les bergers qu'à la fin du XIXe siècle et il est porté essentiellement par les femmes des montagnards les plus riches (Filip 1994 : 17-31). Aujourd'hui ces costumes traditionnels masculins et féminins sont encore portés par tout le monde à Istebna, surtout comme tenues de fête aux grandes occasions. Personne ne porte plus de costume traditionnel au travail. Cette manière de s'habiller pour les fêtes constitue-t-elle une revanche sur le temps ou sur la société ? Nous ne le savons pas, mais nos musiciens essaient à tout prix de démontrer qu'ils ont une histoire, un passé très riche et une identité. Ils savent parler d'eux et cela les rend importants. Ils guettent souvent nos réactions et on a l'impression qu'ils veulent qu'on leur adresse des compliments... Ils sont fiers de leurs origines et de leur particularisme. Il y a aussi un autre point important concernant les origines des montagnards silésiens, mais qui n'est pas évoqué par les autochtones. Il s'agit de l'arrivée des Ruthènes, originaires de la Galicie austro-hongroise, qui, en suivant l'itinéraire des Valaques, longèrent les Carpates et formèrent un groupe de montagnards ukrainiens appelés les Lemkowie. Ceux-ci s'installèrent, entre autres, dans le Beskide silésien (Domaradzka $1997: 410)^{2}$. 
12 Mais la façon de vivre des montagnards silésiens ainsi que leurs motivations pour la musique nous permettent de mieux les connaitre.

\section{La façon de vivre des montagnards silésiens}

\section{Motivations des musiciens montagnards}

\section{Pourquoi font-ils de la musique?}

19 A travers leurs propos, nous saisissons que les montagnards silésiens font de la musique pour cinq raisons principales : c'est un héritage, ils aiment bien faire la fête, notamment pour des occasions comme les mariages, ils conservent des traditions liées aux coutumes saisonnières, ils sont fiers de leur statut de musicien, et ils gagnent parfois de l'argent en jouant.

Héritage: «C'est notre musique et on la fait de père en fils » nous dit Zbigniew Walach, l'un des musiciens d'Istebna. Le montagnard silésien adore la musique, surtout la sienne, et il en est fier. Il la transmet exclusivement de manière orale, comme il l'a entendue et comme on la lui a enseignée. Ceci est très important pour lui et il tient à s'excuser du fait qu'il ne connaît pas les notes de musique et qu'il ne peut pas les écrire pour nous les montrer. En effet, les personnes âgées ne savent pas écrire, mais les jeunes vont à l'école. Ils y apprennent à lire et à écrire le polonais, mais le dialecte de Cieszyn et les patois

Cahiers d'ethnomusicologie, 11 | 2011 
locaux, dont celui d'Istebna, sont exclusivement de tradition orale. Le même phénomène concerne la musique. Les autochtones écoutent bien sûr des cassettes et des musiques à la radio et à la télévision, mais ils n'en disent rien. La tradition musicale locale reste orale à l'image de leur langue. D'ailleurs, l'enseignement des musiques traditionnelles en Pologne n'est pas institutionnalisé. Il n'y a pas de sections de musiques traditionnelles dans les conservatoires et les musiciens apprennent encore dans les campagnes l'art de la musique avec un maitre. Le fait de conserver une tradition orale tout en sachant écrire, indique clairement un rapport fort de la population locale au passé. Cela s'explique peut-être par des raisons historiques ${ }^{3}$. On a toujours exigé de cette population une très grande capacité d'adaptation. Les occupations successives de la région pendant des siècles, obligèrent les gens à être au moins bilingues ${ }^{4}$ (Bazielich 1975 : 27). A la campagne il fallait parler la langue de la famille, à laquelle s'ajoutait celle de l'administration et celle de la paroisse. En ville, celle du travail et, pour les intellectuels, la langue littéraire (Domaradzka 1997 : 243). Aujourd'hui cette microsociété silésienne connaît une évolution, qui reste néanmoins lente, compte tenu des conditions géographiques qui ne favorisent pas des échanges réguliers avec le reste de la région ou même avec les voisins montagnards des autres villages qui, bien sûr, « ne sont pas comme eux » (Gorski 1975).

21 Fête: "Sans musique il n'y a pas de fête et il n'y a pas de mariage » explique Czeslaw Weglarz. On manque de distraction au pays, surtout en hiver, donc on fait de la musique pour se faire plaisir. Sans musique il n'y a pas de fête, surtout celle du mariage. Les déplacements du cortège nuptial de la maison des parents de la jeune mariée vers l'église et ensuite, le plus souvent, vers la salle des fêtes sont accompagnés par des musiciens. Ce ne sont pas toujours les mêmes car cela dépend de la famille dans laquelle on se marie. Mais ils jouent toujours les nuty ou les spiywki à la demande des invités qui les payent. La spiywka est un chant très court, une sorte de refrain qui se compose uniquement d'une phrase, ce qui correspond à un distique. Le point culminant de la cérémonie de mariage est la mise de la coiffe à la jeune mariée, signe de l'appartenance sociale au cercle des femmes mariées. A cette occasion on chante, on danse et on joue toutes les spiywki que le village connaît. On enchaîne plusieurs spiywki à la suite, en fonction de la mémoire des gens qui les interprètent (Bulka 1993). Pour la fin de la fête de mariage, on invente des paroles souvent drôles, voir satiriques.

Tradition: «Il y a aussi d'autres occasions de faire de la musique. Il y a «les nobles », les mojki et aussi la fête des bergers et la moisson. C'est important de garder la tradition ", nous précise Zuzanna Kawulok. En effet, nous avons pu vérifier qu'il existait encore quelques coutumes saisonnières précieusement entretenues, parmi lesquelles certaines ont un rapport direct avec la musique. Les chanteurs de Noël nommés ici les bergers ou les Rois Mages parcourent les villages en étant déguisés et présentent leurs vœux dans toutes les maisons. C'est aussi dans le même but que les «nobles", les «gueux » et les " juhas ", accompagnés des appeaux piszczalki sans trou latéral, organisent des cortèges, où l'on chante et l'on danse en traversant le village (Bazielich 1975 : 153). Dans le dialecte local, le mot «juhas» veut dire : assistant du chef berger. La piszczalka était d'ailleurs l'unique instrument autorisé par l'église avant les Pâques. Plus tard dans l'année, on dit adieu à l'hiver et on souhaite la bienvenue au printemps, en noyant la poupée de paille nommée la marzanna. Mais la tradition du Beskide silésien diffère de celle du reste de la région. Ici, la poupée n'est ni noyée ni brûlée, ce qui correspond à une coutume païenne interdite par l'église catholique et qui a survécu dans toute la Silésie, même dans certains quartiers des grandes villes, telles Wroclaw. Dans le Beskide silésien, les jeunes filles 
fabriquent une poupée en chiffons vêtue d'un costume montagnard, avec laquelle elles parcourent les maisons, où elles reçoivent de l'argent pour leurs chants. Ensuite, elles gardent cette poupée (Bazielich 1975 : 154-155). Les mojki, expression venant du mot maj, qui veut dire «mai », est en rapport avec la fête du mois de mai ayant lieu du 30 avril au 1er mai. Les jeunes gens placent des sapins ornés de rubans et de bonbons devant la maison de leur bien-aimée. Cela provoque parfois, à cause des rivalités, des surprises et des bagarres dont tout le village est au courant. A cette occasion, on chante des chants d'amour et de séduction.

Pour la Pentecôte et le 24 juin, on décore les maisons avec des feuillages, c'est la fête des bergers. A cette occasion, on tresse des couronnes de pissenlits. Les plantes médicinales cueillies à cette date ont un pouvoir magique. On interprète des chants de bergers, nommés helokanie. Il s'agit de très courtes formules de salutation chantées sur les sommets des montagnes. A la fin de la moisson on organise une grande fête au village. On y joue, on y chante, on y danse et on fait surtout des vœux pour la prospérité et la fertilité de la terre. Des croyances et des superstitions liées aux phénomènes de la nature sont toujours bien présentes en Silésie. Des pratiques magiques, héritées des vieux cultes panthéistes préchrétiens sont censées d'assurer une bonne récolte, éviter la grêle ou l'inondation (Bohdanowicz 1995 : 51-61). La vie des montagnards dépend entièrement des caprices de la nature. Il n'y a pas d'assurance d'aide en cas de problèmes, sauf les voisins. D'ailleurs, le caractère du montagnard moyen correspond à son environnement. Il se sent libre, ses comportements sont imprévus. Il est rude, têtu et parfois bagarreur. Mais il sait travailler très durement et il est très efficace dans toutes les activités qu'il entreprend. Quand il a un projet en tête, cela devient une idée fixe, et c'est aussi le cas dans les relations entre hommes et femmes. Il n'échange pas d'alliances à l'église (Dygacz s.d. : 49). Il reste un homme libre, conditionné par la nature et peu influencé par le christianisme et la civilisation des plaines et des villes (Krop $1985: 2)^{5}$.

Fierté: «Nous sommes fiers d'être musiciens car on nous sollicite souvent pour des fêtes ", dit Jacek Kotarba, le violoneux. En effet, les instrumentistes du village ne sont pas très nombreux par rapport aux chanteurs et aux danseurs. Ce sont des privilégiés dont on parle avec admiration. On les envie aussi car ils viennent souvent de familles très connues dans la région et ils perpétuent ainsi activement une tradition familiale. Ils rivalisent entre eux et il est rare que les musiciens vantent les mérites de leurs voisins concurrents. Ils ne parlent que d'eux. D'ailleurs, il faut les rencontrer séparément pour les connaître et savoir quelles sont leurs spécialités. Une motivation complémentaire s'ajoutent aux précédentes. Les manifestations estivales organisées pour les touristes leur plaisent beaucoup. Ils se sentent valorisés et admirés sur scène par les gens venus d'ailleurs qui ne connaissent pas la culture locale. Ils s'estiment importants car ils ont quelque chose à dire, un message à transmettre.

Argent: Une autre motivation s'y ajoute encore, même si les musiciens en parlent moins. Dans le contexte actuel, faire de la musique devient aussi une démarche lucrative. Pendant qu'ils sont sur scène, leurs familles vendent des produits locaux. Se développent ainsi la menuiserie, la gravure sur bois, la tonnellerie; mais on privilégie aussi la sparterie et les arts décoratifs. On se sert de matériaux comme le cuir, le métal et le bois sculpté à chaud. Les foires locales sont pleines d'objets comme des paniers en osier, des chaussures en cuir (kierpce) et des vêtements traditionnels. Il y a aussi les célèbres dentelles de Koniakow, les plus originales de la région (Kazmierczak 1985: 6). Dans le Beskide silésien, il existe surtout des chambres d'hôte plus qu'une hôtellerie bien 
organisée. Il arrive cependant aux musiciens de gagner de l'argent en jouant dans les quelques hôtels des environs.

\section{Comment font-ils de la musique?}

Pour aborder cette question nous donnerons quelques indications concernant les modes d'expression privilégiés par la population locale et la manière d'interpréter la musique.

Modes d'expression: Dans le Beskide silésien, les pratiques vocales et instrumentales sont liées à la danse. Elles se complètent, se substituent ou apparaissent en alternance. Pour nos informateurs, il s'agit « de la même chose ». On opère ici par l'incipit qui sous-entend un distique. Quand on l'énonce, certains le jouent, d'autres le chantent et l'entourage danse car il correspond aussi à des figures de danse. Ce mélange de mélodies vocales, instrumentales et de danse, pour une occasion précise est propre à la tradition du pays ${ }^{6}$. Interprétation: Les chants s'interprètent en position assise, accroupie, debout et en mouvement. Cela dépend des occasions et des endroits. Il peut s'agir de fêtes en plein air sur une scène montée à cet effet ou d'une fête de bergers en pleine nature. D'autres coutumes, comme celle du mariage, se passent à l'extérieur, mais aussi à l'intérieur de la maison. Il y a encore l'habitude du travail collectif domestique et saisonnier (dentellerie, bricolage, moisson) agrémenté de musique. Pour prolonger la musique dans le temps, on utilise les procédés de répétition et de collage au niveau des paroles et des musiques. Cela provoque souvent une hétérophonie car les musiciens jouent leurs différentes versions en même temps. Des décalages rythmiques, assortis d'une forte expressivité, sont le symbole même du montagnard type, spontané et déchaîné dans son rude élément ${ }^{7}$, surtout quand il interprète des polkas devenant fantaisistes, au tempo irrégulier et au rythme libre.

Le montagnard silésien fait aussi le choix de certaines thématiques.

\section{Que chantent-ils?}

Les montagnards interprètent aujourd'hui volontiers des chants d'amour, mais ils connaissent encore des chants de travail (Tacina 1939) et certains chants de coutumes (Kopoczek 1984). Leurs paroles décrivent une société assez fermée, ce qui ne veut pas dire rigide, rigoriste ou puritaine, où les mœurs paraissent, au contraire, assez libres. Il s'agit majoritairement de "paroles de femmes » sur leurs bien-aimés (Domaradzka 1997 : 512-603). Celui-ci peut être éleveur (berger), agriculteur (laboureur) ou artisan. On y évoque la nature et la médecine par les plantes. La femme, le plus souvent une jeune fille, y exprime ses attentes et ses craintes concernant son amoureux. Ces chants de mariage l'avertissent des difficultés de sa future vie d'épouse, la préparant ainsi à franchir une étape difficile. Il s'agit d'un rite de passage. Même si on y trouve une réflexion générale sur la vie, par exemple sur les violences conjugales ou sur les tromperies, les questions que se pose la jeune fille - «m'aime-t-il réellement, m'aimera-t-il toujours? » - restent essentielles.

\section{Que disent-ils de leur musique?}

Les témoignages des montagnards silésiens seront présentés autour des notions de patrimoine, de passé, de fabrication, de jeu, de complexe, concernant les instruments 
traditionnels, autour de leurs propres expressions sur la musique et avec un regard sur les manifestations locales.

Patrimoine: Ils adorent parler de leurs instruments de musique, qu'ils conservent avec beaucoup de soin, car il s'agit pour eux d'un patrimoine. C'est aussi un savoir-faire car ils ont appris les secrets la fabrication avec leurs pères. Cette tradition familiale est très ancienne dans le Beskide silésien. Aujourd'hui, ce sont surtout les familles d'Istebna qui la perpétuent avec le plus de succès.

Passé: Les montagnards silésiens ont deux façons de se rattacher volontairement au passé: premièrement, par l'intermédiaire de leurs instruments de musique dont ils aiment décrire les fonctions d'origine ; en second lieu, par leurs récits sur le passé dont la fonction est d'éduquer les jeunes.

La trombita, un type de trompe joué en plein air, d'une longueur pouvant atteindre 4,5 m., est encore installée dans les huttes de berger et dans les maisons à Brenna, Istebna (Antoni Kawulok) et à Wisla (Andrzej Szalbot). Elle permettait la communication à distance, souvent pour avertir d'un danger ou lancer un appel au secours à l'approche de voleurs. Par contre, le cor de berger, aux dimensions plus réduites $(2,5 \mathrm{~m}$.), servait à émettre des signaux pour chasser le loup qui s'approchait du troupeau. Ces instruments sont la fierté de la population locale et on les installe sur les scènes estivales lors de la Semaine de la Culture du Beskide. Le rôle de la flûte piszczalka de Szalasnik était initialement d'accompagner la transhumance. Présente dans les huttes de berger nommées szalasy, d'où son nom, elle change ensuite de fonction. On l'utilise pour agrémenter des soirées de travail en commun organisées du mois de décembre jusqu'au printemps. Les travaux tels écosser, plumer et faire la dentelle sont accompagnés de chants et de récits. Le travail une fois terminé, des fêtes dansantes sont organisées massivement avant le Carême. La fujarka (un mirliton), une feuille de sureau et un brin d'herbe permettent l'interprétation de mélodies « pour faire paître les vaches ». Zuzanna Kawulok nous en a joué plusieurs. Les plus jeunes du village qui viennent régulièrement chez elle en connaissent aussi. 
Fig. 2: Transmission du jeu de la piszczalka à huit trous, par Zuzanna Kawulok, au musée des instruments hérités de son père, à Istebna

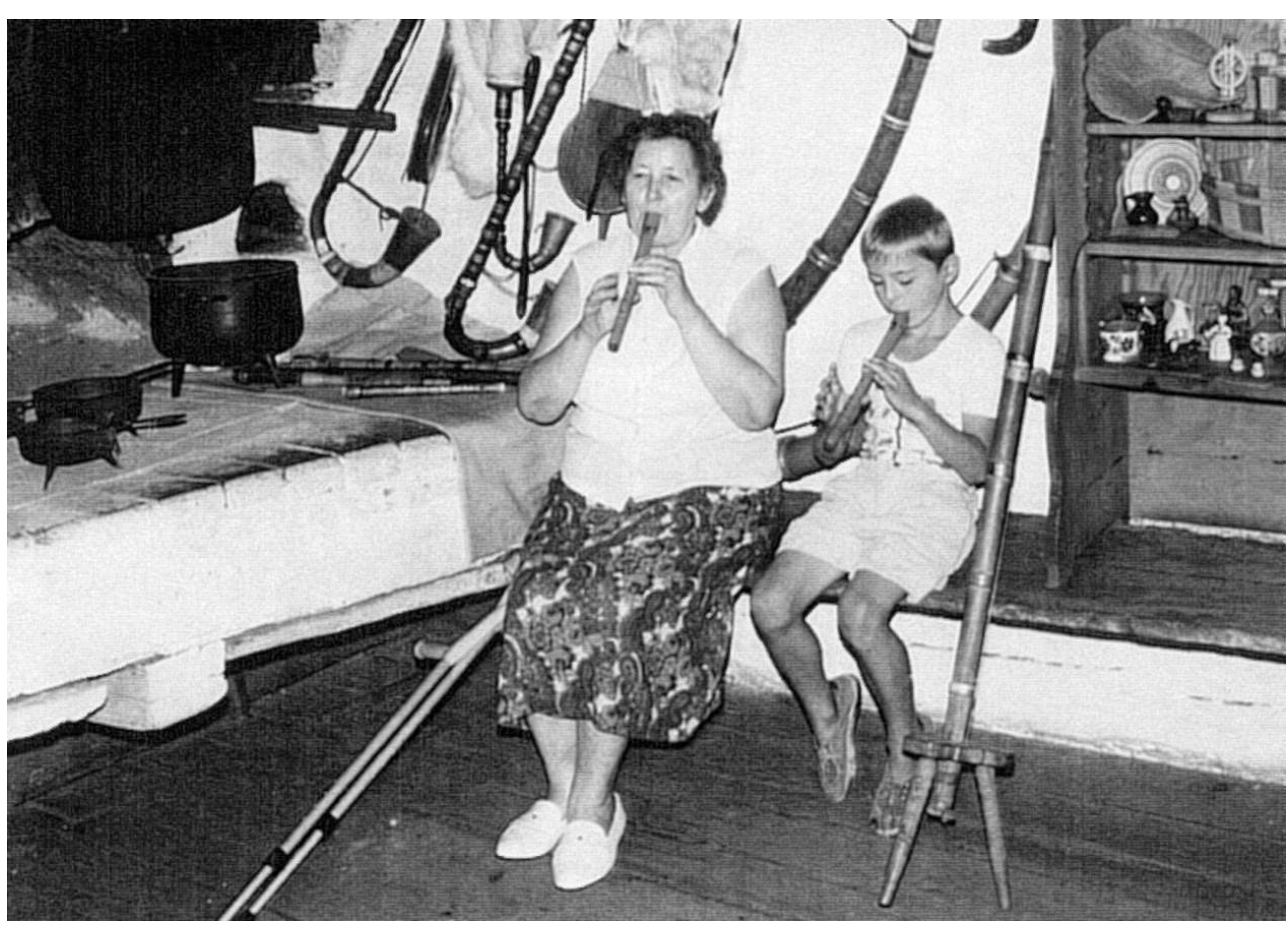

Photo: Aurélia Domaradzka Barbier

Zuzanna Kawulok est aussi poétesse et sait bien s'exprimer. Pour cela elle utilise le piszczek. une sorte d'appeau, et sa musique improvisée traduit ses émotions. Il sert à rendre plus expressifs ses récits anciens, souvent à morale. Son but est d'éduquer les nouvelles générations. Elle aime bien raconter l'histoire d'un petit garçon qui rêvait d'une galette chaude, mais qui devait surveiller les vaches en étant à jeun, car le travail passait avant toute chose. Il s'agit pour elle d'un récit exemplaire et le plus important est de retenir que le travail passait en priorité, même pour les enfants. Elle a constaté, non sans une certaine amertume, qu'aujourd'hui les parents n'agiraient plus ainsi.

Fabrication: Nous avons pu assister à certaines phases de construction, de réglage et d'accordage de l'ocarina, de la cornemuse gajdy et des cordophones. On nous a aussi expliqué les problèmes d'entretien de ces instruments. Dans les maisons montagnardes d'Istebna on trouve une multitude d'outils servant au bricolage, mais aussi à l'ébauche des instruments de musique tant des plus simples - sifflets de toutes sortes, piszczalki et fujarki - que des plus complexes, ceux du duo local traditionnel : le gajdy et le violon. La fabrication d'un ocarina peut se faire en plein air car elle n'exige pas beaucoup de temps ni de place.

Zbigniew Walach a toujours des cornes de vache bien sèches dans sa cave. Il prévoit les emplacements des trous en fonction de la morphologie de ses mains. A l'intérieur, il ajuste le bois en le raclant; il travaille ensuite sur le débit d'air et des sons obtenus pour apporter les modifications finales. La construction du gajdy est beaucoup plus complexe. Il faut aussi remarquer que les ornements métalliques étaient depuis longtemps une des spécialités d'Istebna, de Koniakow et de Cisowica car les mines y étaient nombreuses. Les instruments transmis par la famille sont extrêmement précieux. Le réglage de ces instruments, choyés et traités avec un soin jaloux, est très difficile. Ils se dérèglent 
facilement à cause des écarts de température. Selon nos informateurs, le secret de leur construction est arrivé ici avec les Valaques. Le gajdy est unique par rapport aux autres cornemuses de Pologne car il est pourvu d'un sac à souffler (dymlok) qui permet de chanter en jouant. La décoration de l'instrument est sophistiquée et l'accordage se fait à l'aide d'un fil ou de cire.

Jeu : La manière de jouer d'un violoneux est surprenante : le violon est placé initialement sur la poitrine, il se déplace sur le bras, sur la hanche ou même verticalement. Il en va de même pour la basy. Le musicien n'hésite pas à s'agenouiller pour en jouer. On en joue aussi en marchant. Aujourd'hui rarement joué, le violon traditionnel en bois de prunier zlóbcoki a un son très criard. On continue à construire des contrebasses de petite taille ( basy). Les musiciens sont aussi préoccupés par le son. Ils décrivent et montrent volontiers les difficultés qu'ils ont pour obtenir un son juste à la piszczalka sans trou latéral et à la piszczalka de Szalasnik. En effet, ces instruments fonctionnant avec des partiels donnent souvent des sons mobiles et parfois instables. Les mélodies jouées sont des formules archaïques, valaques et montagnardes. Les cors de bergers et les trombity nécessitent un entretien régulier. Pour en jouer avec succès, il faut d'abord les laisser se gonfler dans l'eau pour éviter les fausses notes dues à des fissures ${ }^{8}$.

Fig. 3: Transmission du savoir-faire de la fabrication d'un ocarina en corne, par Zbigniew Walach, chez lui à Istebna

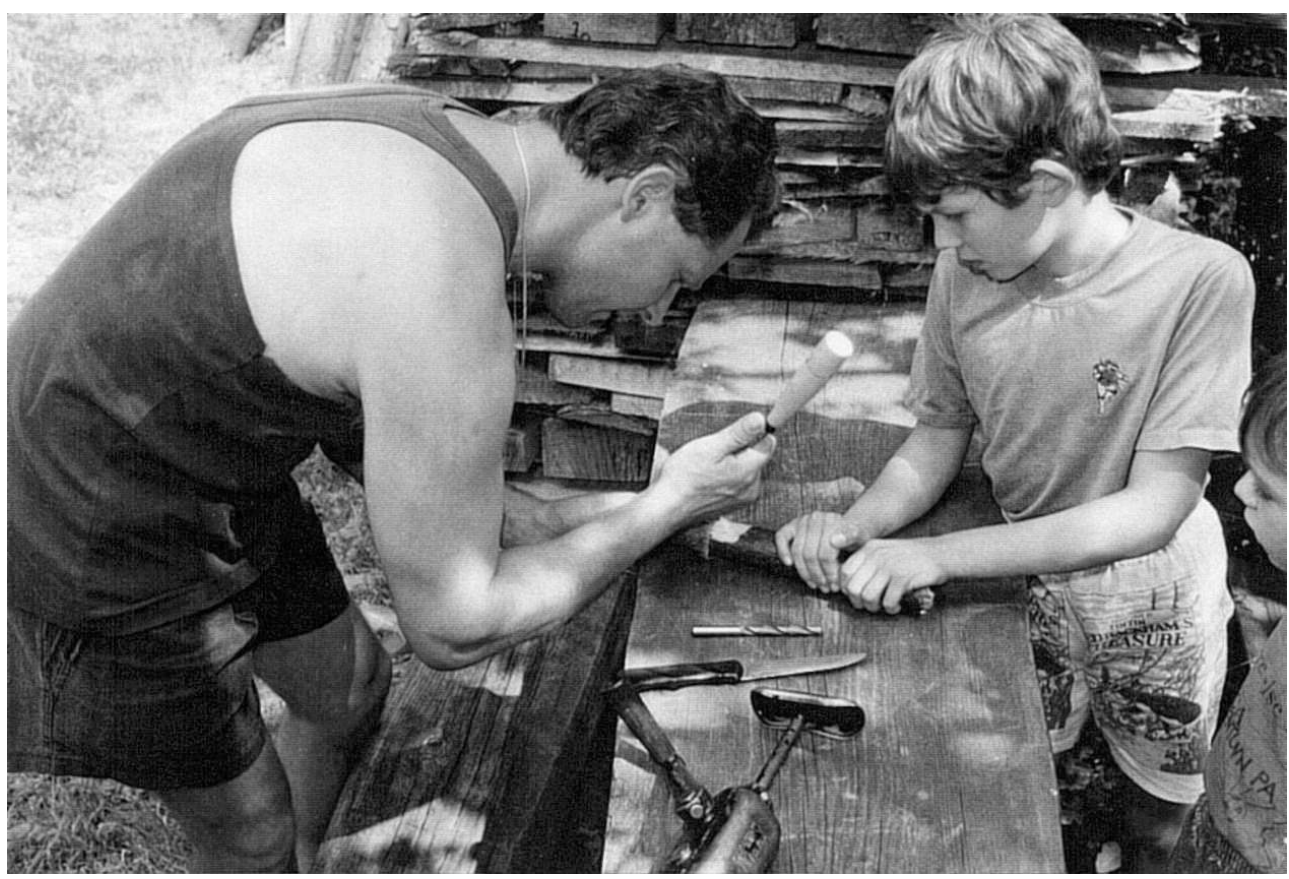

Photo: Aurélia Domaradzka Barbier

Complexe: Malgré un patriotisme local et la volonté d'être mieux que les autres, qui ressort aussi dans l'existence de rivalités entre les familles, on ne dit pas ici que l'on joue parfaitement. Il y a dans cette société un complexe concernant la musique tsigane qui, pour eux, est plus virtuose que la leur. Dans le temps, les orchestres montagnards des auberges ont été supplantés par des groupes de Tsiganes de passage, qui ravissaient l'auditoire par leur technique. Pour cette raison, nos informateurs ont cru indispensable de donner une explication et même de se justifier. Selon eux, le peuple local ne peut pas avoir de musiciens sachant jouer très vite car ils vivent essentiellement de la terre; ils 
ont les mains détériorées par un dur travail manuel, donc des mains peu agiles. De plus, faire de la musique n'est pas un métier pour eux, comme c'est le cas pour les Tsiganes, mais une occupation après le travail. Il faut aussi savoir qu'à partir du XVIe siècle, se développe en Silésie la tradition des musiciens ambulants.

Expressions : Nos informateurs soulignent le fait qu'ils ont appris leurs chants oralement et qu'ils transmettent ces chants aux jeunes de la même façon. La transmission se fait donc par l'écoute et l'imitation accompagnées de la parole. En dehors d'un vocabulaire de base qui définit les nuty (les spiywki), c'est-à-dire les chants, on parle ici de chants «très anciens ", valaques. Ces formules " très anciennes » se caractérisent par la présence de la seconde augmentée. Quand l'accompagnement de ces chants se fait à l'orchestre (la basy, le gajdy, le prym et le sekund) cela crée des décalages rythmiques et une hétérophonie. Quand ils emploient l'expression: "la musique de chez nous», il s'agit de chants montagnards incluant la quarte augmentée. Certains chants «très anciens» ont de nombreuses variantes car chacun privilégie les sonorités et les instruments qui lui conviennent le mieux. Pour cette raison, on utilise l'expression : « à la manière de... ». Par exemple le chant connu de tous intitulé Doliny, doliny, doliny ("Vallées, vallées, vallées ») est perçu par Jozef Broda comme homogène, il l'interprète en utilisant la seconde augmentée comme élément unificateur. La version de Zbigniew Walach, selon lui la plus ancienne qui existe, se compose d'une formule "très ancienne» et d'une formule montagnarde. Une autre version «à la manière de Vieux Colombus d'Andziolowka » est aussi homogène, mais de style exclusivement montagnard (la présence de la quarte augmentée). Une version moderne d'Istebna est construite sur une formule en mineur et sur une formule "très ancienne ». Il existe aussi des expressions en rapport avec des formules fixes d'accompagnement du début et de la fin du chant. Elles sont surtout associées au gajdy qui fait partie du duo traditionnel local.

41 Transmises de génération en génération, elles sont adaptées en fonction des circonstances. Le secret pour mémoriser les sons de ces formules nous a été communiqué par Zbigniew Walach, qui dit qu'il faut les mémoriser sur les paroles suivantes : «zdaloby sie cos wypic» (« il serait bon de boire quelque chose »). Zbigniew Walach et son groupe nous ont aussi présenté des chants en tchèque, dont le plus connu est Oci, oci (« Les yeux, les yeux »). Mais, ils nous ont dit que, de l'autre côté de la frontière, on connaît les chants de chez eux. Rien d'étonnant, compte tenu de leur histoire commune et mouvementée (Hlawiczka 1975 : 11-63).

Manifestations : La participation à des manifestations musicales dans un village plus grand, à Wisla, nous a permis de nous rendre compte que certaines traditions familiales ont disparu avec la mort des grands-parents, surtout entre 1970 et 1980. Les familles musiciennes sont donc moins nombreuses dans les villages du "triangle du Beskide ». On peut en rencontrer sur les scènes régionales montées chaque année pendant quelques jours à Wisla, à Szczyrk, à Zywiec, à Makow Podhalanski et à Oswiecim, village par ailleurs universellement et dramatiquement connu sous son nom allemand d'Auschwitz. Une trentaine de groupes locaux y participent ${ }^{9}$. Ces groupes comptent autant des gens âgés que des enfants. Les noms des groupes viennent d'un nom de famille ou de localité, ce qui indique deux sortes de traditions : familiale et villageoise. A cette occasion, il faut aussi remarquer que l'existence de la Semaine de la Culture du Beskide correspond bien à un besoin de la population locale, tant culturel qu'économique, et non pas à une directive ministérielle imposée dans le cadre du développement du folklorisme. Selon nos informateurs de Wisla, la Semaine de la Culture du Beskide est née, en quelque sorte, par 
jalousie car une fête semblable était organisée annuellement, à partir de 1950, par les montagnards silésiens installés à Jablunkov en République tchèque. Les habitants de Wisla considéraient qu'il était impensable que les «Polonais » vivant hors frontières arrivent à s'organiser, contrairement aux gens du pays. La grande Fête des Montagnes de 1938 était encore présente dans l'esprit des informateurs les plus âgés. Ils indiquaient aussi que c'est Stefan Pojda, habitant de Wisla, qui, dès 1964 et pendant trente ans, conduisit le cortège solennel vers la scène. Nous avons aussi appris qu'en 1964, seuls huit groupes se produisirent sur scène, dont "Wisla ", "Koniakow " et "Jaworzynka ». D'autres informateurs se sont rappelés qu'à partir de 1966 on se produisait aussi à Szczyrk. A partir des années 1970 on installe des estrades à Zywiec, à Makow Podhalanski et dans d'autres localités voisines. En 1980 à Ujsoly, à la veille de la St Wawrzyniec, on n'hésite pas à allumer des feux de plusieurs mètres sur les pentes des montagnes environnantes, pour y chanter et danser. A partir de 1990, ces manifestations acquièrent une dimension internationale. Dès 1993, on organise une journée exclusivement consacrée aux groupes des bords du fleuve Olza, région nommée la Zaolzie. En 1994, la scène accueille quatre-vingt-un groupes et trois mille artistes. Ces rencontres musicales sont financées par le Ministère de la Culture et le Conseil Régional. Contrairement à la scène de Zywiec, ville célèbre pour sa bière, qui privilégie la notion de compétition, car le jury attribue là-bas de multiples prix dans les catégories d'ensembles de chanteurs ou de danseurs, d'orchestres traditionnels, de chorales populaires, de chanteurs solistes et d'instrumentistes: le meilleur maître et son apprenti, le but des autres scènes locales reste ludique. C'est aussi l'occasion de montrer les plus beaux et les plus anciens costumes de fête qui font la fierté des familles.

Examiner la question relative aux dimensions cognitives et extramusicales de la musique traditionnelle d'une région montagnarde d'Europe Centrale, implique la prise en compte d'un contexte géopolitique complexe, donc d'une dimension historique, sans en omettre la dimension ludique et économique. Pour les musiciens du Beskide silésien, issus d'une population montagnarde à l'identité forte et aux traditions très vivantes, la musique est l'une des formes privilégiées d'affirmation de leur identité aussi bien collective qu'individuelle. La distinction entre les deux est d'ailleurs fragile tant leur ego plonge ses racines dans leur identité collective. Les notions de transmission héréditaire, de «très ancien » et de tradition sont fondamentales, même si ces traditions sont parfois beaucoup plus récentes que les montagnards ne l'imaginent. La fête, qu'elle soit liée aux cérémonies familiales ou au cycle annuel, est le point de jonction du plaisir et de la tradition, et la musique est selon eux sa meilleure expression. Le musicien montagnard reconnaît cependant que la pratique musicale est aussi le moyen de gagner un peu d'argent, toujours bienvenu dans une région montagnarde où la vie est difficile. Mais, fondamentalement, la musique est pour lui une manière d'affirmer sa supériorité et celle de sa famille. Là encore, sa supériorité résulte non seulement de son propre talent, mais aussi de la tradition héritée de ses pères. Le spectateur étranger au village apporte non seulement son argent, mais surtout sa reconnaissance et son hommage au musicien fier de son héritage. 
Fig. 4 : Groupe de Zbigniew Walach d'Istebna : le duo traditionnel du Beskide silésien, gajdy et violon, se transforme ici en trio, avec la présence du zlobcoki

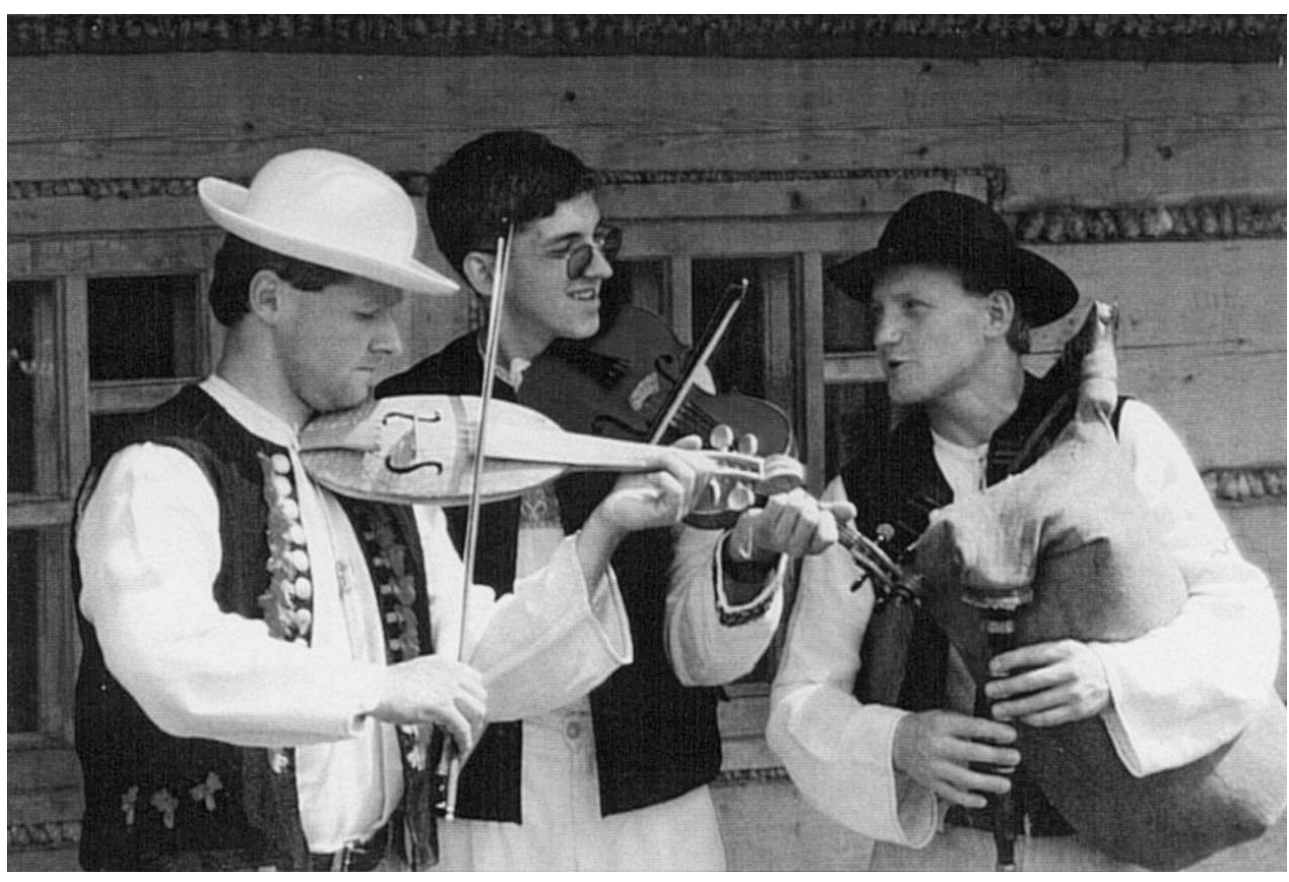

Dans un contexte d'analyse systémique des problèmes posés, on est tenté de proposer un organigramme relationnel entre les modes d'expression et les composantes ethnologiques subordonnées. Il est évident que l'ensemble des relations de dépendance dépasse largement le cadre du présent travail.

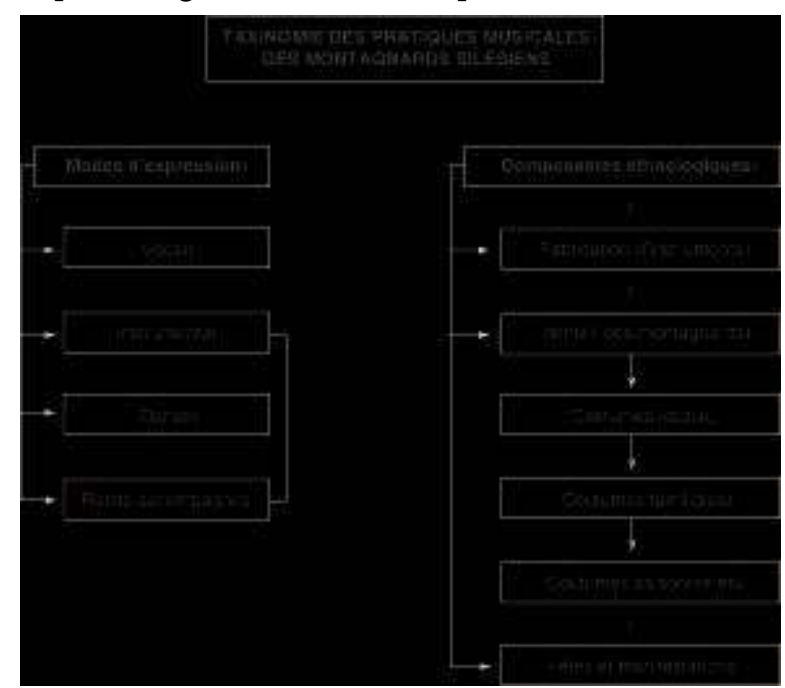

On constate que dans le Beskide silésien, région isolée à forte identité, on s'appuie sur le respect des coutumes et de la tradition, sans oublier la dimension économique. Sa musique traditionnelle a évolué et comme toute autre musique, elle subit des transformations. Cependant, malgré la mondialisation générée, entre autres, par les grands circuits de diffusion, la musique traditionnelle développée par les montagnards du Beskide silésien garde son style formulaire, ses mélodies archaïques, ses rythmes libres et ses couplets très courts intégrés dans les modes d'expression syncrétiques. Quand aux 
paroles des musiciens, elles exacerbent cette spécificité, en valorisant l'individu dans sa tradition familiale et régionale.

\section{BIBLIOGRAPHIE}

BAZIELICH Barbara, 1975, Zabytki ludowej kultury na Slaski i w Czestochowskim. Bytom : PLT.

BOHDANOWICZ Janusz, 1995, Demonologia ludowa. Katowice : Literatura Ludowa ${ }^{\circ} 1$.

BULKA Wladyslaw, 1993, Goralskie spiewki weselne. Zywiec : Boomex.

CHYBINSKI Adolf, 1907, O metodach zbierania i porzadkowania piesni. Lwow : Towarzystwo Ludowe. DOMARADZKA Aurélia, 1997, Le folklore musical polonais : la Silésie. Contribution à l'étude du chant populaire silésien. Thèse de Doctorat. Lyon : Université Lumière Lyon 2.

DYGACZ Adolf, s. d., Zwyczaje i taniec in Lud cieszynski. Wroclaw : Ossolineum.

FILIP Elzbieta Teresa, 1994, Nie szata zdobi czlowieka - Wystawa etnografia. Bielsko-Biala : Uni Art. GORSKI Ryszard, 1975, Co wies to inna piesn. Wroclaw: P.A.N.

HLAWICZKA Karol, 1976, Przenikanie i wzajemne wplywy elementow muzycznych polskich i czeskich w folklorze cieszynskim in Z zagadnien folkloru muzycznego na Slasku. Katowice: Uniwersytet Slaski.

KAZMIERCZAK Maria, 1985, Istebna, Jaworzynka, Koniakow. Katowice: KAW.

KOPOCZEK Alojzy, 1984, Instrumenty muzyczne Beskidu Slaskiego i Zywieckiego. Bielsko-Biala: BTSK.

KROP Jan, 1985, Istebna, Jaworzynka, Koniakow. Katowice: KAW.

PREIZNER Tadeusz, 1938, Piesni ludowe w Beskidzie Slaskim. Katowice : Slaskie Wiadomosci Muzyczne

SELLIER André et Jean, 1991, Atlas des peuples d'Europe centrale. Paris : La Découverte.

SIKORA Wladyslaw, 1993, Cieszynska piesn i poezja ludowa i ich zwiazki interetniczne. Katowice : Literatura ludowa nos4-6.

SKRZYPCZAK Andrzej, 1996, Dziedzictwo kulturowe : informacja, mniejszosci etniczne. Warszawa : SBP.

TACINA Jan, 1939, Gronie nasze gronie. Katowice : SLASK.

\section{NOTES}

1. Le mot "berger" ne doit pas être pris ici au sens français de simple gardien de troupeau, souvent un peu misérable, prolétaire de l'élevage. En Europe Centrale et Balkanique, comme dans d'autres pays de peuples pasteurs, le berger est le propriétaire du troupeau, donc le maître de la richesse essentielle de la communauté. Il est riche et il le montre. 
2. Entre le XVe et le XVIIIe siècles, des expansions polonaises à l'est vers l'Ukraine ont lieu et cela provoque le phénomène de "rutenizacja», terme traduit dans les sources ethnologiques polonaises par « ruthenisation ", dans le sens de " russification » ce qui n'est pas exact. Il s'agit des Ruthènes, en particulier les Lemkowie, montagnards ukrainiens installés dans le Beskide silésien, dans le Beskide Sadecki et en Podhale. On suppose que les Lemkowie furent à l'origine de religion greco-catholique et influencés par la culture byzantine.

3. Malgré son morcellement au XII ${ }^{\mathrm{e}}$ et au XIII ${ }^{\mathrm{e}}$ siècles, la Pologne parvient presque à se reconstituer en royaume en 1295, mais à l'exception de la Silésie. Au début du XV siècle, Vladislas, le fils du roi de Pologne Casimir le Grand, élu roi de Hongrie, reconstitue le royaume de Bohême avec la Silésie (Sellier 1991 : 107). En 1526, la Silésie et la Bohême se retrouvent sous la tutelle autrichienne des Habsbourg. Ainsi « le triangle du Beskide» devint une zone frontalière de la Hongrie, dont les frontières arrivèrent au Jablunkovsky Prusmyk. La Silésie restera donc au cœur des querelles de suzeraineté et des échanges entre les grandes familles souveraines d'Europe Centrale, polonaises, tchèques et hongroises et plus tard autrichiennes et allemandes.

4. Dwutorowosc jezyczna - terme proposé par Barbara Bazielich pouvant se traduire par "phénomène linguistique à double voie ".

5. Ces villages furent longtemps d'accès très difficile. La première église catholique fut construite à Istebna seulement en 1720 ; elle dut son existence aux effets de la Contre-Réforme. Son but était de convertir le peuple montagnard au catholicisme. C'est un jésuite, Leopold Tempes, travaillant pour l'évêché de Wroclaw en Basse Silésie qui fut le vecteur de cette politique dans les montagnes. Mais jusqu'au XIX ${ }^{\mathrm{e}}$ siècle les montagnards vécurent en ayant très peu de contacts avec la ville.

6. Ce phénomène de syncrétisme - fusion de la musique, de la parole et de la danse a déjà été remarqué dans la culture montagnarde des Carpates (Chybinski 1907: 13). On y a noté l'indépendance des discours verbal et musical.

7. On raisonne ici par phrases. Chacune constitue une entité à part entière. Enchaînées à la suite, elles sont entrecoupées de silences plus au moins longs et expressifs. En fin de phrases les valeurs rythmiques deviennent lentes, emphatiques comme dans le folklore tchèque et surtout hongrois

8. Suite aux analyses des variantes des chants silésiens que nous avons effectuées, nous pouvons aussi constater que ces chants ne s'exportent pas dans le reste de la région, et cela à cause de leurs particularités mélodiques et rythmiques dues à l'emploi des instruments locaux et à leur interprétation très personnalisée. Par contre, les chants qui arrivent ici du reste de la Silésie et des régions voisines sont « traduits » à la manière locale.

9. Les groupes actuels du district de Bielsko Biala sont les suivants : Babiogorcy de Zawoia, Beskid de Bielsko-Biala, Brenna de Brenna, Czantoria de Leszna Gorka, Czantoria d'Ustron, Fil de Wilamowice, Gilowianka de Gilowice, Gronicek de Gilowice, Jozef Szczotka de Milowka et de Wegierska Gorka, Jaworze de Jaworzno, Kety de Kety, Klimczok de Szczyrk, Koniakow de Koniakow, Kotarzanie de Brenna, Magurzanie de Lodygowice, Male Kety de Kety, Maly Koniakow de Koniakow, Ondraszek de Szczyrk, Polana Makowska de Makow Podhalanski, Porabczanie de Porabka, Rajcusie de Rajcza, Solanka de Sol, Szarotki de Kamesznica, Wadowice de Wadowice, Wisla de Wisla et Ziemia Cieszynska de Cieszyn. 


\section{RÉSUMÉS}

Les musiciens du Beskide silésien, petite région montagnarde située au sud de la Silésie en Pologne, sont marqués par la culture des Carpates. Leur identité est très forte et ils attachent beaucoup d'importance dans leurs propos à la tradition qu'ils imaginent "très ancienne ", transmise par leurs pères. Pour eux, la fête ne va pas sans musique dont ils ont une conception syncrétique ; il s'agit d'un mélange de mélodies vocales, instrumentales et de danse. La musique, indépendamment de l'argent qu'elle permet de rapporter grâce aux touristes, est surtout un moyen de reconnaissance sociale, d'affirmation de sa supériorité individuelle, familiale et régionale. Leurs discours sur leurs instruments de musique, leur fabrication, la difficulté de les accorder et d'en jouer, est aussi un moyen de se singulariser et de se valoriser. Enfin, leurs témoignages mettent en avant la dimension sociale de la musique, à la fois dans sa création et dans sa pratique.

Musicians from Beskydy, which is a small mountainous region situated in the southern part of Silesia in Poland, have been deeply influenced by Carpatian culture. They have a strong sense of identity and attach a great deal of importance to what they regard as the ancient traditions handed down by their forefathers. For them, there is no celebration without music of which they have a syncretic conception. What we are referring to here, is a mixture of vocal melodies, instrumentals and dance. Apart from income stemming from the tourist trade, music is mainly a source of social recognition, of individual, family and regional «superiority». Discourse regarding their instruments, their making, the complexities of tuning them, the difficulty of playing them well, are all ways of enabling individuals to stand out from the crowd and of increasing the esteem in which they themselves are held. Finally, their testimonies highlight the social role and importance of music both in its creation and performance.

\section{AUTEUR}

\section{AURÉLIA DOMARADZKA BARBIER}

Aurélia Domaradzka Barbier, née en 1964 en Silésie (Pologne), est arrivée en France en 1984.

Parallèlement à des études de musique au Conservatoire et de musicologie à l'Université Lumière Lyon 2, elle a continué son activité d'auteur, compositeur, interprète en donnant des concerts et en participant à des émissions de radio et de télévision en Pologne et en France. Elle enseigne la musique et l'histoire des arts dans un lycée lyonnais. Sa thèse de doctorat a été consacrée à la musique silésienne. De nouveaux concepts et de nouveaux outils d'analyse y ont été avancés dans un contexte ethnologique. A présent, sa recherche porte sur les cultures montagnardes européennes, parallèlement à des recherches sur la voix, effectuées dans le cadre de l'A.P.M.C. de l'Université Lumière Lyon 2 et du Centre de Recherche C.E.F.I. de l’Université de Savoie. 